'Unidad de Hematología Clínica Hospital del Salvador. ${ }^{2}$ Instituto Nacional del Tórax ${ }^{a}$ Equipo de trasplante.

Recibido el 29 de agosto de 2014, aceptado el 18 de noviembre de 2014

Correspondencia a: Dra. Camila Peña O. camipena@gmail.com

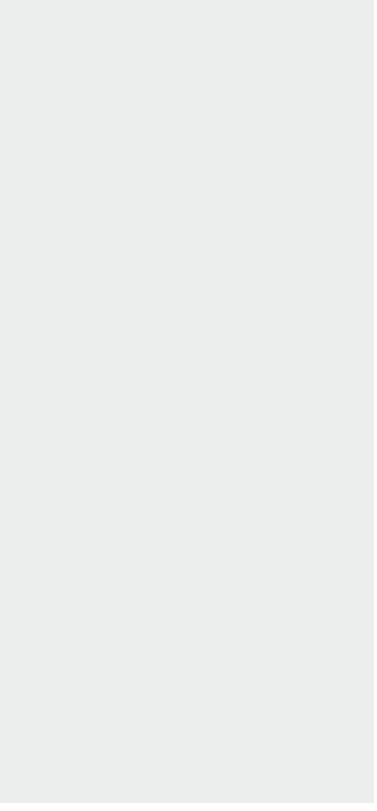

\section{Leucemia mieloide aguda secundaria a inmunosupresores en una paciente trasplantada de pulmón, una inusual y letal entidad}

\author{
CAMILA PEÑA ${ }^{1}$, CLAUDIA SEPÚLVEDA ${ }^{2, a}$, \\ JOEL MELO $^{2}$, CAROLINA GUERRA $^{1}$
}

\section{Secondary acute myeloid leukemia in a lung allograft recipient. Report of one case}

Secondary acute myeloid leukemia is a very rare complication in patients with solid organ transplantation. We report a 62 years old female who received a right single lung allograft for idiopathic pulmonary fibrosis. Her immunosuppression scheme consisted in prednisone, azathioprine, and tacrolimus. Two years after the transplantation, she presented with progressive pancytopenia. Bone marrow aspiration was informed as a M4 acute myeloid leukemia, confirmed by flow cytometry. Cytogenetic study was complex, including alterations in chromosome 5. A secondary acute myeloid leukemia was diagnosed. The patient developed nosocomial pneumonia and died a few days after the diagnosis, without specific treatment. The pathogenesis of acute myeloid leukemia is probably related to the intensive exposure to immunosuppressant, especially azathioprine, in these patients.

(Rev Med Chile 2015; 143: 262-265)

Key words: Leukemia, myeloid, acute; Lung transplantation; Immunosuppressive agents.
E l trasplante de pulmón es la única alternativa terapéutica en enfermedades pulmonares terminales. El aumento de la sobrevida gracias a los avances en inmunosupresión ha llevado consigo un aumento de las complicaciones como son las neoplasias malignas. Las más frecuentes son la enfermedad linfoproliferativa post trasplante en $21 \%^{1}$, las neoplasias cutáneas, el sarcoma de Kaposi y otros carcinomas ${ }^{2,3}$. La leucemia aguda es una manifestación neoplásica muy rara, con una incidencia aproximada de 0,2 a 2,5\% de los trasplantados ${ }^{4}$, y cuya aparición guarda relación a la mayor sobrevida de estos pacientes y por ende mayor exposición a inmunosupresores. Se presenta el caso de una paciente trasplantada de pulmón que evolucionó con una leucemia aguda secundaria a inmunosupresión.

\section{Caso clínico}

Paciente mujer de 62 años que fue sometida a trasplante monopulmonar derecho en mayo de 2011 por una fibrosis pulmonar idiopática. Al tercer mes, se diagnosticó una neumonía criptogénica y un tromboembolismo pulmonar bilateral, por lo que recibió bolos de corticoides y anticoagulación por 6 meses, evolucionando en forma satisfactoria. $\mathrm{Su}$ esquema de inmunosupresión consistía en prednisona, azatioprina y tacrolimus. En profilaxis con cotrimoxazol, itraconazol y aciclovir. En controles posteriores sólo destacó anemia leve permanente, pero con función pulmonar y radiología normal. Desde julio de 2013 se agregó progresivamente leucopenia, por lo que se suspendió cotrimoxazol y se recontroló con hemograma, que 


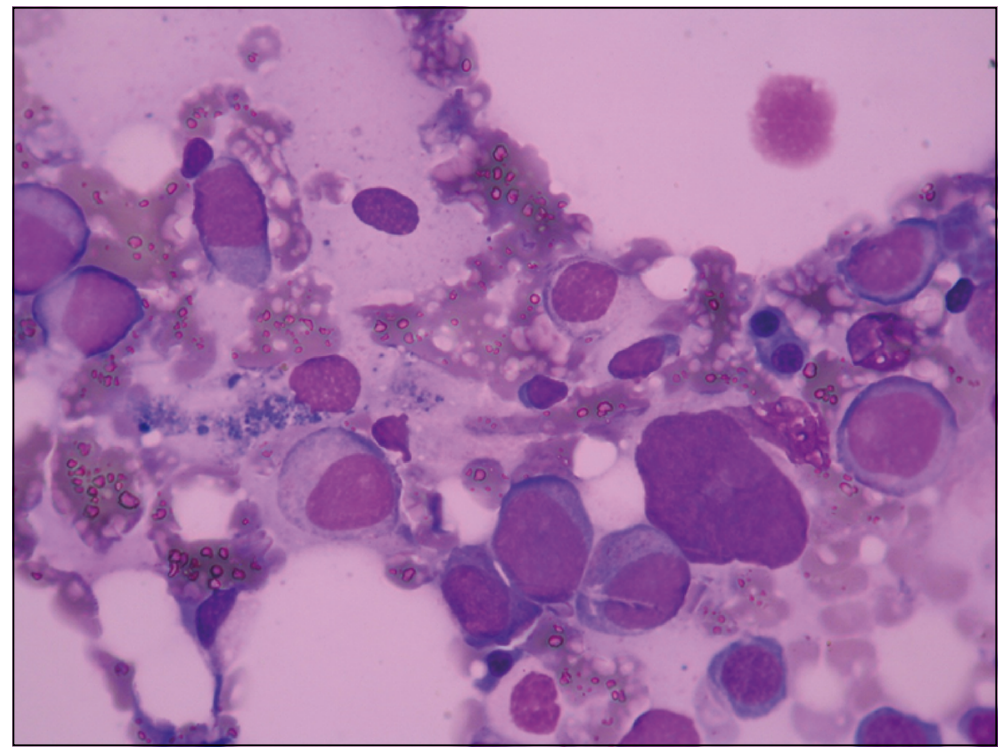

Figura 1. Mielograma de la paciente. Se observa blastos y displasia en las demás células. Tinción May Grunwald Giemsa. Aumento 100X. mostraba: $\mathrm{Hb} 11 \mathrm{~g} / \mathrm{dL}$, leucocitos de $2.000 / \mathrm{mm}^{3}$, neutrófilos de $400 / \mathrm{mm}^{3}$ y plaquetas normales. Se pensó en neutropenia secundaria a fármacos, por lo que se suspendió la azatioprina. Se dejó además con ácido fólico, vitamina B12 y dado que la paciente evolucionó con un cuadro infeccioso y con mayor disminución de neutrófilos, se indicó un estimulador de colonias de granulocitos. Sin embargo, la paciente evolucionó con pancitopenia progresiva. Hemograma de control a la semana fue: $\mathrm{Hb} 6,8 \mathrm{~g} / \mathrm{dL}$, leucocitos $500 / \mathrm{mm}^{3}$, neutrófilos de $100 / \mathrm{mm}^{3}$ y plaquetas de $15.000 / \mathrm{mm}^{3}$, con algunos signos de displasia en los neutrófilos. Se realizó mielograma que mostró $20 \%$ de blastos mieloides (Figura 1). Se atribuyó en un comienzo a la recuperación de la médula ósea, sin embargo, en un frotis de control se encontró $2 \%$ de blastos, por lo que se realizó un nuevo mielograma. Este mostró 35\% de blastos de abundante citoplasma, de aspecto monocitoide. El estudio inmunofenotípico mostró blastos de estirpe mieloide, de los cuales, 5\% correspondieron a FSC/SSC bajo, CD45 débil, que expresan CD34, CD13, CD15, CD33, CD64, CD117 y CD19; y 8\% de monocitos patológicos displásticos, FSC/SSC intermedio, CD45 moderado, que expresan CD34, CD13, CD15, CD33, CD64, HLA-DR y CD19, además de 40\% de granulocitos inmunofenotípicamente displásticos. Por citogenética se analizaron 27 metafases con bandeo G, 7 de ellas cariotipadas, encontrándose un clon anormal hiperdiploide de 58 60 cromosomas, con trisomías de los cromosomas $6,8,10,14,15$, y 20 , y tetrasomías del 11 y 21 . También se encuentra un cromosoma 1 extra con deleción del brazo q, y un cromosoma 5 con una deleción intersticial del brazo q en duplicado. Se elaboró un cariotipo compuesto por la heterogeneidad celular: 58 60,XX,+X,+del(1) (q10), del(5) (?q13q33),+del(5)(?q13q33),+6,+8,+10,+11,+11, $+13,+14,+15,+20,+21,+21$. Se concluyó leucemia mielomonocítica con displasia granulocítica. Las alteraciones complejas junto con alteraciones del cromosoma 5 observadas sugirieron una leucemia aguda relacionada a terapia. La paciente evolucionó con falla ventilatoria secundaria a neumonía intrahospitalaria de rápida evolución y falleció finalmente a los pocos días.

\section{Discusión}

La leucemia aguda asociada a trasplante de órgano sólido es muy infrecuente. Tanto así, que en un principio se dudó de su relación con el trasplante y se creyó que correspondían a leucemias agudas al azar. Sin embargo, hay estudios que comprobaron el aumento de su incidencia en este tipo de pacientes ${ }^{5,6}$. 
Se ha descrito en casos en trasplante de hígado, corazón y riñón. En pacientes con trasplante pulmonar ha sido descrita con mucho menor frecuencia y sólo someramente dentro de estudios que incluyen leucemias agudas en todos los tipos de trasplantes de órganos sólidos ${ }^{5,7,8}$. Cabe destacar además que en 2 revisiones publicadas sobre neoplasias secundarias en trasplante pulmonar específicamente, no se encontró ninguna leucemia mieloide aguda (LMA) ${ }^{9,10}$.

Debido a lo raro de esta entidad, no se conoce con claridad sus características. La incidencia de LMA en trasplante de órgano sólido se evaluó en un estudio de Offman et al que reunió los datos de más de 170.000 pacientes 5 . En este se encontró 37 pacientes con LMA de 31.691 trasplantados de corazón, pulmón o ambos, es decir, $0,1 \%$ de los pacientes. El riesgo relativo comparado con controles sanos según edad, sexo y origen geográfico fue de 5,5 para trasplantados cardiacos o pulmonares. En general fue mayor en hombres (un 89\% de las LMA en trasplante cardiaco/pulmonar), sin embargo, hay que hacer notar que la mayoría de los trasplantados eran de sexo masculino. Una reciente revisión sistemática de Rashidi et $\mathrm{al}^{11}$ en que se evaluó todos los casos de LMA post trasplante reportados en la literatura hasta su publicación, se vio una edad media de 50 años, aunque mayor en trasplante pulmonar, y con un intervalo desde el trasplante a la LMA de 3,8 años, la mayoría M4/ M5 (39\%) y con sobrevida global de 3 meses. Todo esto es compatible con nuestro caso. Cabe destacar que en esta revisión sólo se encontraron 2 casos de LMA en trasplante pulmonar. En general, se describe la aparición de LMA después del tercer año post trasplante, aunque se han descrito entre 2 meses a 17 años post trasplante ${ }^{8}$. Esto podría diferenciar esta patología de los linfoma no Hodgkin relacionados a terapia, cuya incidencia es mayor en el primer año post trasplante, lo que sugiere diferentes mecanismos en su fisiopatología.

Como se expuso anteriormente, la mayor sobrevida de estos pacientes los ha llevado a una mayor exposición a fármacos potencialmente leucemogénicos. De hecho los inmunosupresores son el factor de riesgo más importante en el desarrollo de neoplasias post trasplante, aunque su mecanismo exacto aún no se conoce ${ }^{12}$. Se cree que sería consecuencia de la disregulación del sistema inmune y de que estas drogas serían capaces de infligir daño al ADN, lo que lleva consigo un aumento de las neoplasias y una disminución en la capacidad de combatirlas por el sistema inmune ${ }^{13}$. En cuanto a qué inmunosupresor está involucrado, según un registro del Cincinnati Transplant Tumor Registry ${ }^{14}$ la LMA se desarrolla en 3\% de los tumores en trasplantados con inmunosupresores "no-ciclosporina". Los pacientes con ciclosporina desarrollan LMA en menos de $1 \%$. Por otro lado, Morton et al demostró que la azatioprina específicamente aumenta el riesgo de síndrome mielodisplásico y de leucemia mieloide aguda, 2 a 5 años posterior al trasplante, lo que concuerda con nuestro caso ${ }^{15}$.

El mismo estudio de Offman ${ }^{5}$ evaluó también el posible rol de la inmunosupresión. El $90 \%$ de los trasplantes de corazón/pulmón recibieron azatioprina y/o ciclosporina. La incidencia de LMA y SMD fue mayor, estadísticamente significativo en los pacientes que usaron 2 a $3 \mathrm{mg} / \mathrm{kg}$ al día de azatioprina comparado con los usuarios de $1 \mathrm{mg} /$ $\mathrm{kg} /$ día. Como dato importante además se nombra que 2 de 5 pacientes con LMA en trasplante cardiaco/pulmón recibieron irradiación total linfoide, lo que lleva a pensar que el riesgo podría aumentar con la radiación.

En cuanto a lo encontrado en laboratorio, y tal como se mencionó previamente, lo más frecuente es la LMA M4/M5, aunque se han descrito todos los tipos de LMA (desde M0 hasta M7). Es interesante recalcar que el segundo tipo más frecuente (25\%) es la leucemia promielocítica aguda, descrita sobretodo secundaria a trasplante hepático ${ }^{16,17}$. Esta leucemia tiene un tratamiento específico y mejor pronóstico que los demás subtipos.

Según la Organización Mundial de la Salud, las neoplasias relacionadas a terapia, que engloban tanto la mielodisplasia como la leucemia aguda secundaria a terapia, se dividen en 2 grupos más frecuentes: las neoplasias secundarias a topoisomerasa II y las secundarias a alquilantes. Dentro de este último grupo se encuentran también las secundarias a radioterapia o a drogas inmunosupresoras ${ }^{18}$. La citogenética descrita más frecuentemente de este grupo son las alteraciones complejas, y especialmente pérdidas en el cromosoma 5 o el 7 y alteraciones del 11q23 (gen MLL). Nuestra paciente presentó blastos mieloides y monocitoides (M4) con signos de mielodisplasia en el mielograma y por citometría de flujo. La citogenética mostró un cariotipo complejo y alteraciones del cromosoma 5, por lo que se asumió LMA secun- 
daria. Otras alteraciones relacionadas con leucemia secundaria, son la trisomía 8 (que también presentó nuestra paciente), monosomía 7 y t (15; 17) y la inv16. Se han descrito casos con trisomía 8 y FLT3 positivo, los que tendrían especialmente mal pronóstico ${ }^{19}$.

Lamentablemente no se realizó FLT3 en nuestra paciente.

El tratamiento de estas leucemias no difieren de las LMA de novo, es decir, quimioterapia intensiva. Sin embargo, estos tipos de LMA tienen peor pronóstico que las LMA de novo, incluso con un diagnóstico precoz y tratamiento adecua$\mathrm{do}^{20}$. Nuestra paciente falleció a los pocos días del diagnóstico por falla ventilatoria secundaria a neumonía intrahospitalaria, sin haber recibido tratamiento para la LMA. Esto concuerda con los estudios que muestran a las infecciones y la leucemia refractaria como las causas más frecuentes de muerte $e^{7,8,17}$.

\section{Referencias}

1. Penn I. Cancers complicating organ transplantation. N Engl J Med 1990; 323: 1767-9.

2. Penn I. Occurrence of cancers in immunosuppressed organ transplant recipients. Clin Transpl 1998; 147-58.

3. Buell JF, Gross TG, Woodle ES. Malignancy after transplantation. Transplantation. 2005; 80: S254-S264.

4. Doti CA, Gondolesi GE, Sheiner PA, Emre S, Miller CM, Aledort LM. Leukemia after liver transplant. Transplantation 2001; 72: 1643-6.

5. Offman J, Opelz G, Doehler B, Cummins D, Halil O, Banner NR, et al. Defective DNA mismatch repair in acute myeloid leukemia/myelodysplastic syndrome after organ transplantation. Blood. 2004; 104 (3): 822-8.

6. Engels EA, Pfeiffer RM, Fraumeni JF Jr, Kasiske BL, Israni AK, Snyder JJ, et al. Spectrum of cancer risk among US solid organ transplant recipients. JAMA 2011; 306 (17): 1891-901.

7. Kourelis TV, Boruchov A, Hull D, Polio J, Scholes J, Moustakakis M, et al. Acute myeloid leukemia following solid organ transplantation: case report and comprehen- sive review. Conn Med. 2012; 76 (3) 151-4.

8. Thalhammer-Scherrer R, Wieselthaler G, Knoebl P, Schwarzinger I, Simonitsch I, Mitterbauer G, et al. Posttransplant acute myeloid leukemia (PT-AML). Leukemia 1999; 13: 321-6.

9. Anile M, Venuta F, Diso D, De Giacomo T, Rendina EA, Rolla M, et al. Malignancies following lung transplantation. Transplant Proc. 2007; 39 (6): 1983-4.

10. Parada MT, Sepúlveda C, Alba A, Salas A. Malignancy Development in Lung Transplant. Patients Transplant Proc 2011; 43 (6) 2316-7.

11. Rashidi A, Fisher SI. Acute myeloid leukemia following solid organ transplantation: entity or novelty? Eur J Haematol 2014; 92 (6): 459-66.

12. Dantal J, Soulillou JP. Immunosuppressive drugs and the risk of cancer after organ transplantation. $\mathrm{N}$ Engl J Med 2005; 352: 1371-3.

13. Leone G, Mele L, Pulsoni A, Equitani F, Pagano L. The incidence of secondary leukemias. Haematologica 1999; 84: 937-45.

14. Penn I, Brunson ME. Cancers after cyclosporine therapy. Transplant Proc 1988; 20 (Suppl 3): 885-92.

15. Morton LM, Gibson TM, Clarke CA, Lynch CF, Anderson LA, Pfeiffer R, et al. Risk of myeloid neoplasms after solid organ transplantation. Leukemia 2014 Apr 14 [Epub ahead of print].

16. Liu M, Liu J, Liu L, Yu L, Shi B, Ye L, et al. A Case Report of Acute Myeloid Leukemia after Liver Transplantation Acta Haematol 2013; 129 (4) 225-8.

17. Cho YU, Chi HS, Park CJ, Seo EJ, Lee JH, Lee JH, et al. Two cases of post-liver transplant acute myeloid leukemia in Korean adults: review of bibliographies and comparison with post-renal transplant acute myeloid leukemia. Ann Hematol 2008; 87: 513-4.

18. Larson RA. Therapy-related myeloid neoplasms. Haematologica 2009; 94 (4): 454-9.

19. Jiang N, Li H, Wang GS, Zhang J, Zhang JF, Yi SH, et al. Acute leukemia, a rare but fatal complication after liver transplantation. Leukemia Research 2009; 33: 1349-51.

20. Kern W, Haferlach T, Schnittger S, Hiddemann W, Schoch C. Prognosis in therapy-related acute myeloid leukemia and impact of karyotype. J Clin Oncol 2004; 22: $2510-1$. 